\title{
On the momentum of solitons and vortex rings in a superfluid
}

\author{
L.P. Pitaevskii \\ P.L. Kapitza Institute for Physical Problems RAS, \\ 119334, Moscow, ul. Kosygina 2, RF; \\ Dipartimento di Fisica, Università di Trento and INO-CNR BEC Center, \\ I-38123 Trento, Povo, Italy.
}

\begin{abstract}
This paper is devoted to the calculation of the momentum of localized excitations, such as solitons and vortex rings, moving in a superfluid. The direct calculation of the momentum by integration of the mass flux density results in a badly-converging integral. I suggest a method for the renormalization of the integral with the explicit separation of a term related to the vortex line. This term can be calculated explicitly and gives the main contribution for the rings whose size is large compared to the healing length. I compare my method with the Jones and Roberts prescription for renormalization. I investigate the case of a uniform superfluid, and that of a superfluid in a cylindrical trap. I discuss the calculation of the jump in the phase of the order parameter and obtain a simple estimate for this jump for a large ring in the trap.
\end{abstract}

PACS numbers: 03.75.Lm, 3.75.Kk, 67.85.De

\section{Introduction}

One of the characteristic properties of a superfluid is the possible existence of localized stationary excitations. These can be solitons, vortex rings, solitonic vortices, or other more complicated objects. The study of the vortex rings 
has resulted in important progress in the understanding of the physics of superfluid helium. A very interesting possibility arose after the creation of new superfluid systems like ultracold Bose and Fermi gases, which are confined in traps. The dynamics of the excitations in these systems is an important theoretical and experimental problem due to the presence of external fields. At the same time, due to the diluteness of the gases, one can develop a microscopic theory which is sufficiently detailed. If the trapping field changes slowly enough, it is convenient to solve the problem in two steps. As a first step, it is reasonable to find the energy and momentum for an excitation in a uniform fluid. (In the case of an elongated trap, the fluid is uniform in the axial direction.) Second, one can analyze the motion of the excitations using the semi-classical equations of motion. Depending on the nature of the problem, the energy should be expressed in terms of momentum or velocity. However, a difficulty arises which had already been encountered in classical hydrodynamics (see for instance [1, §11] or [2, ch. 6]). If one tries to calculate the momentum by integration of the mass flux density $\mathbf{j}$ over the volume of a fluid, i.e. using the equation:

$$
\mathbf{p}=\int \mathbf{j} d^{3} x
$$

then, for stationary motion, the integrand does not decrease fast enough and the value of the integral depends on the shape of the integration domain. Different prescriptions have been suggested to overcome this difficulty. If one knows the energy $\varepsilon$ as a function of the excitation velocity $V$, the momentum can be calculated by integrating the following equation:

$$
(\partial \varepsilon / \partial p)=V
$$

This method was used in the Landau-Lifshitz book [1]. However, it does not allow for the definition of the integration constant, which is important for the calculation of the Landau critical velocity. In his book [2], Batchelor assumed that the moving body was initially at rest and subsequently set in motion by an external force, and he calculated the momentum which was transferred to the body and the fluid (see ch. 7). The same method was used to calculate the momentum of a classical vortex ring. Roberts and Grant [3], and Jones and Roberts [4], used a similar approach to calculate the momentum of a vortex ring in a Bose-Einstein condensate (BEC), using the Gross-Pitaevskii (GP) equation. In Ref. [3], the authors considered a ring whose radius is large 
compared to the healing length $\xi$. In Ref. [4], a ring with arbitrary radius was considered, and a general renormalized equation for the momentum was obtained in the form of an absolutely convergent integral. I shall discuss it below. The existence of such an expression is related to the fact that a perturbation propagates in a fluid with a finite velocity, which does not exceed the velocity of sound. Because the excitation was formed at some initial time, this means that at large enough distances the velocity of flow is zero. There is, however, a difficulty, because at these distances the problem is not stationary. In this article I suggest a different renormalized equation for the momentum. This equation is convenient for the calculation of the momentum of an excitation which contains a quantized vortex of large size, because the main term related to the vorticity is separated explicitly. I shall assume that the superfluid is described by an order parameter $\Psi$. Its phase $\phi$ defines the superfluid velocity according to the equation $\mathbf{v}=\frac{\hbar}{M} \nabla \phi$, where $M=m$ for a bosonic superfluid and $M=2 m$ for a fermionic superfuid, and $m$ is the atomic mass. For simplicity, I shall also assume that the mass flux density is equal to

$$
\mathbf{j}=\rho \mathbf{v},
$$

with $\rho$ being the mass density of the fluid. This equation is licit in the context of the GP equation and several of it generalizations. (In the general case, $\mathbf{j}$ can also depend on the derivatives of $\mathbf{v}$.) However, this last assumption is not necessary. It is only important that Eq. (3) be always satisfied at large distances, where hydrodynamics is valid. One can present the density as $\rho=\rho_{\infty}+\delta \rho$, where $\rho_{\infty}$ is the unperturbed density at large distances. Then the momentum will be presented as:

$$
\mathbf{p}=\frac{\hbar}{M} \int \rho_{\infty} \nabla \phi d^{3} x+\frac{\hbar}{M} \int \delta \rho \nabla \phi d^{3} x
$$

The second integral converges absolutely, because the density perturbation $\delta \rho$, being quadratic in $\mathbf{v}$, decreases fast. The first term can be calculated in a general form, taking into account that the phase is equal to zero at infinity. Note that this method was used in [1, §65] for the calculation of the momentum of a wave packet of sound waves. I shall consider below several examples of the application of Eq. (44) in order to demonstrate the effectiveness of the method. 


\section{An instructive example: Induced momen- tum of a sphere moving in a fluid.}

As a first example, I shall consider the classical problem of the calculation of the momentum of a sphere of radius $R$ moving with the velocity $\mathbf{V}$ in an incompressible liquid. We shall see that the problem can be solved by the suggested method in a surprisingly simple way. The second term in Eq. (4) is equal to zero due to the incompressibility of the liquid. The first term can be transformed into an integral on the infinitely remote surface, which is equal to zero, and an integral over the surface of the sphere. As a result one gets for the momentum of the liquid:

$$
\mathbf{p}_{f l}=-\rho \frac{\hbar}{M} \oint \mathbf{n} \phi d \sigma,
$$

where the vector $\mathbf{n}$ is normal to the surface and $d \sigma$ is an element of the surface. (It is called the induced momentum.) Note that the integral over the remote surface being zero implies that the liquid is still compressible and that the remote surface is on the distances $r>c t$, where $c$ is the speed of sound, $t$ is the time from the initial moment of motion. However, if the velocity of motion is much smaller than the speed of sound, $V \ll c$, then one can neglect the compressibility on distances of the order of $R$ and a solution of the hydrodynamic problem gives the potential for the velocity on the surface:

$$
\frac{\hbar}{M} \phi=-\frac{R}{2} \mathbf{n} \cdot \mathbf{V} .
$$

In this case, the momentum is

$$
\mathbf{p}_{f l}=\frac{2 \pi}{3} R^{3} \rho \mathbf{V},
$$

in accordance with Eq. (11.6) in [1] (see also Eq. (6.4.29) in [2]).

\section{Vortex rings in a uniform fluid}

Let us now consider an excitation moving with a constant velocity $\mathbf{V}$ in an infinite volume of a uniform fluid. Consider first an excitation which does not contain vortex lines. For instance, it can be the wonderful Jones-Roberts soliton. A vortex ring degenerates into this excitation when its velocity 
approaches $V=0.88 c$ [4]. (In the 2D case an analytical solitonic solution was constructed by Manakov et al [5].) Then the first integral in Eq. (44) can be transformed in a surface integral over the remote surface, which is equal to zero, and the momentum of the excitation is:

$$
\mathbf{p}=\frac{\hbar}{M} \int \delta \rho \nabla \phi d^{3} x
$$

However, if there is a loop of a quantum vortex in the structure of the excitation, the situation is different. In this case, the phase $\phi$ is not a single-valued function of the coordinates: it acquires an additional $2 \pi$ when going around the vortex line. Now, in order to apply the Gauss theorem, one should perform a cut. The values of $\phi$ on the two sides of the cut differ by $2 \pi$. This makes $\phi$ single--valued. One can choose as a cut any surface which closes the aperture of the loop. Now, besides the remote surface, whose contribution is zero, one should integrate over both sides of the cut. We finally get for the momentum:

$$
\mathbf{p}=\rho_{\infty} \frac{2 \pi \hbar}{M} \oint \mathbf{n} d \sigma+\frac{\hbar}{M} \int \delta \rho \nabla \phi d^{3} x .
$$

The integration in the first term is performed over the surface of the cut, the vector $\mathbf{n}$ is normal to this surface, and $d \sigma$ is an element of the surface. This equation is the main result of the present paper. In the case of a circular ring, moving along the $x$ direction, the momentum is:

$$
p=\rho_{\infty} \frac{2 \pi^{2} \hbar}{M} R^{2}+\frac{\hbar}{M} \int \delta \rho \nabla_{x} \phi d^{3} x .
$$

An important property of this expression is that for a large ring, whose radius $R$ satisfies

$$
R \gg \xi
$$

with $\xi \sim \hbar / m c$ being the healing length of the fluid, the two terms in Eq. (10) have different orders of magnitude. Indeed, a simple estimate shows that the second term is of the order of $\rho_{\infty} \hbar \xi^{2} / M$, that is, much smaller than the first term 1 . Thus, the momentum of the large ring is given by the first term in Eq. (10). This equation is well known. It was used to describe the hydrodynamics of a classical liquid. Feynman used it when discussing

\footnotetext{
${ }^{1}$ It is important for the estimate that, under the condition (11), the velocity of the ring $V \ll c$.
} 
the critical velocity related to the creation of the vortex rings [6]. However, this equation is difficult to derive starting from a microscopic theory such as the GP equation (see the discussion in [3]). Using the suggested method, this problem is easily solved. Jones and Roberts used a different method to renormalize the equation for the momentum of a vortex ring of arbitrary radius on the basis of the GP equation. They subtracted the integral

$$
\frac{\hbar}{2 i M} \sqrt{\rho_{\infty}} \int\left[\nabla \Psi-\nabla \Psi^{*}\right] d^{3} x=-\frac{\hbar}{M} \sqrt{\rho_{\infty}} \int \nabla(\sqrt{\rho} \sin \phi) d^{3} x
$$

from the general equation (11) for the momentum. (Recall that, in the GP equation, $\Psi=\sqrt{\rho} e^{i \phi}$.) This integral is equal to zero, because, unlike $\phi$, the order parameter $\Psi$ is a single-valued function of the coordinates, and the integral is transformed into an integral over the remote surface only, where $\phi=0$. They finally obtained the following expression for the momentum:

$$
\mathbf{p}=\frac{\hbar}{2 i M} \int\left[\left(\Psi^{*}-\sqrt{\rho_{\infty}}\right) \nabla \Psi-\left(\Psi-\sqrt{\rho_{\infty}}\right) \nabla \Psi^{*}\right] d^{3} x
$$

The authors of Ref. [9] used this equation to calculate the momenta of solitons, vortex rings and solitonic vortices. The integral (13) is absolutely convergent, but it is difficult to simplify this equation in the case of a large ring, because one should find a corresponding asymptotic equation for $\Psi$. An approximate wave function which is accurate enough was constructed by Berloff, see [7, Eq. 25]. Using this function, one can calculate the momentum analytically according to Eq. (13). In the limit $R \rightarrow \infty$, one gets $\rho_{\infty} 2 \pi^{2} \hbar R^{2} / M$ [8], in accordance with Eq. (10), as it should be.

\section{Solitons and vortex rings in a cylindrical trap.}

A natural method for the experimental investigation of the dynamics of the excitations is the observation of their motion along the axis of an elongated trap. As I have explained above, the first step to develop the theory of such a motion is to calculate the energy and the momentum of the excitation in the trap. My goal is to calculate the momentum. I shall use the same considerations as in the case of a uniform fluid. However, the situation in a cylindrical trap has its peculiarities. For simplicity, I shall consider a fluid in 
an axially-symmetric potential. Let us call $x$ the coordinate along the axis and $r$ the radial coordinate. It is not difficult to show that the velocity field decreases fast enough at large distances $|x|$ from any stationary excitation. Thus the integral in Eq. (1) converges absolutely. Let us call $p_{s}$ its result. It seems that there no longer is any motivation for the renormalization of the momentum. However, this is not the case. The point is that the stationary solution for the order parameter has a phase jump between $x=-\infty$ and $x=+\infty$ :

$$
\Delta \phi=\phi(x=\infty)-\phi(x=-\infty)
$$

Nevertheless, for an excitation created at $t=0$, there is no perturbation of the phase at infinity. This means that, at large enough distances from the excitation, there arises a counter-current which compensates the phase jump. If the region of the counterflow is long enough, the velocity $\bar{v}$ of the fluid in the counterflow is small and the counterflow does not contribute to the energy, but it does contributes to the momentum 2 . Let $\rho_{\infty}(r)$ denote the density of the fluid at large distances $|x| \rightarrow \infty$. Then, the contribution of the counterflow to the momentum is:

$$
\Delta p=\int \rho_{\infty} 2 \pi r d r \frac{\hbar}{M} \int \partial_{x} \phi d x=-\hbar \rho_{1 \infty} \Delta \phi / M
$$

where the integral with respect to $x$ is taken over the region of the counterflow, and $\rho_{1 \infty}=\int \rho_{\infty} 2 \pi r d r$ is the 1D density of the unperturbed fluid. Note that the result (15) does not depend on the velocity distribution in the counterflow region or on its length. Thus the momentum is [10]:

$$
p=p_{s}+\Delta p=\frac{\hbar}{M} \int \rho(x, r) \partial_{x} \phi d^{3} x-\rho_{1 \infty} \frac{\hbar}{M} \Delta \phi .
$$

In this equation, the quantities $\rho$ and $\phi$ should be calculated assuming that they depend on $x$ and $t$ through the combination $x-V t$ (stationary motion). The integral over $x$ is taken from $-\infty$ to $\infty$. As I have already said, it converges under the accepted assumption. Note that adding to the phase an integer multiple of $2 \pi$ does not change the state of the system. This means that values of the momentum which differ by a multiple of $2 \pi \hbar \rho_{1 \infty} / M$ are physically equivalent. This is a general property of a quantum fluid in a 1D geometry. This fluid can be considered as a 1D periodic structure with

\footnotetext{
${ }^{2}$ It is possible to show (see [9]) that $\phi( \pm \infty)$ does not depend on $r$. Correspondingly, the velocity $\bar{v}$ does not depend on $r$ either.
} 
the period $M / \rho_{1 \infty}$, smeared by quantum fluctuations, and $p$ has properties of the quasimomentum [11. One can obtain a different expression for the momentum, using the general Eq. (4). Calculating the surface integral as in the previous section, we find for a circular vortex ring:

$$
p=\frac{2 \pi \hbar}{M} \int_{0}^{R} \rho_{\infty}(r) 2 \pi r d r+\frac{\hbar}{M} \int \delta \rho \nabla_{x} \phi d^{3} x .
$$

An advantage of this equation, analogously to equation (10), is that in the case of a large ring, $R \gg \xi$, the first term of this equation is much larger than the second, which can be neglected. (This situation occurs only in the Thomas-Fermi limit, where $R_{\perp} \gg \xi$, with $R_{\perp}$ being the radial size of the cloud.) Equation (17) was derived and used in my paper [12].

\section{Estimate of the phase jump.}

The phase jump $\Delta \phi$ which we considered in the previous section is an interesting physical quantity. It can be measured by observing the interference pattern in the fluid expansion which occurs after the trap is switched off. Of course, if one can calculate the order parameter around the excitation, one will know the phase jump. However, it is interesting to estimate the jump for a large-sized vortex ring, that is, $R \gg \xi$, in a cylindrical trap. Let us equalize the equations (16) and (17) for the momentum:

$$
\Delta \phi=-\frac{2 \pi}{\rho_{1}} \int_{0}^{R} \rho_{\infty}(r) 2 \pi r d r-\frac{1}{\rho_{1}} \int \delta \rho \nabla_{x} \phi d^{3} x+\frac{M}{\hbar} p_{s} .
$$

One can transform the quantity $p_{s}$ in this equation exploiting Galilean invariance. Let us consider the mass flux density integrated over the cross-section of the trap, $j_{1}^{(0)}=\int j_{x} d y d z$, in the coordinate frame moving with the velocity of the ring. In this frame, the density of the fluid does not depend on time. Then, from the continuity equation $\partial_{t} \rho_{1}+\partial_{x} j_{1}^{(0)}=0$, it follows that $j_{1}^{(0)}$ does not depend on the coordinate and is equal to its value at large distances, where the $1 \mathrm{D}$ density is equal to the unperturbed value $\rho_{1 \infty}$ and the velocity of fluid is $-V$. Thus $j_{1}^{(0)}=-\rho_{1 \infty} V$. Now, the Galilean transformation gives $j_{1}(x)=j_{1}^{(0)}+\rho_{1}(x) V=\delta \rho_{1} V$ for the current density in the laboratory frame. Integration with respect to $x$ yields $p_{s}=V \int \delta \rho_{1} d x$. The 
quantity $M_{s}=\int \delta \rho_{1} d x$ represents the mass of the excitation. Substituting this expression into Eq. (18) gives $\Delta \phi$ :

$$
\Delta \phi=-\frac{2 \pi}{\rho_{1}} \int_{0}^{R} \rho_{\infty}(r) 2 \pi r d r+\frac{1}{\rho_{1}} \int \delta \rho\left(\frac{M}{\hbar} V-\nabla_{x} \phi\right) d^{3} x .
$$

It is obvious that for a large ring the second term in this equation is small and can be neglected. Then, the phase jump is given by the simple equation:

$$
\Delta \phi \approx-2 \pi \frac{\rho_{1 R}}{\rho_{1}}
$$

where $\rho_{1 R}=\int_{0}^{R} \rho_{\infty}(r) 2 \pi r d r$ is the $1 \mathrm{D}$ fluid density contained in a cylinder of radius $R$. It would be interesting to check this equation in numerical simulations of the GP equation, analogous to [9], but for a vortex ring with $R_{\perp} \gg \xi$. Note that it is not difficult to generalize the derived equations for a geometry which does not present axial symmetry, when in the trap there exists a vortex line which ends on the border of the sample. (I am considering the Thomas-Fermi limit, in which case the boundary is sharp.) In this case the cut should be drawn from the line to the boundary. Such a situation occurs for the solitonic vortex [9, 13, 14, 15].

In conclusion, I have presented in this paper a convenient method for the calculation of the momentum of localized excitations in superfluids. The method reduces the problem to an absolutely-convergent integral, and it is particularly convenient in the presence of vortex lines of large length.

I thank P.H. Roberts for useful comments on the paper [4], N.G. Berloff for discussions and the calculation of momentum according to the wave function [7], S. Komineas for sending data from the calculations of Ref. [9], D.J. Papoular, T. Yefsah and I.A. Fomin for discussions.

This work was supported by the ERC through the QGBE grant, the Provincia Autonoma di Trento, and the Italian MIUR through the PRIN2009 grant.

I am glad that the present paper will be published in the special issue of JETP devoted to the 75-th birthday of A.F. Andreev. I have had the possibility to familiarize myself with the work of this outstanding physicist from the very beginning of his scientific carrier. In my recollection, A.F. Andreev has succeeded to solve actually any problem he has tackled. His papers are always based on the deep understanding of the physical meaning of a phenomenon and extraordinary clearness of thinking. They are very elegant and 
have often stimulated new experiments. I always read his articles with pleasure and great benefit for myself. We are grateful to A.F. that he sacrifices a part of his precious time for scientific management activities, trying as much as he can to save our science. I congratulate my friend and wish him permanent desire to work and good health - to him and his wonderful family. The rest is a matter of course.

\section{References}

[1] L.D. Landau, E.M. Lifshitz, Fluid Mechanics, Pergamon Press, Oxford (1987).

[2] G.K. Batchelor, An Introduction to Fluid Dynamics, Cambridge University Press, Cambridge (1970).

[3] P.H. Roberts, J. Grant, J. Phys. A: Gen. Phys. 4, 55 (1971).

[4] C.A. Jones, P.H. Roberts, J. Phys. A: Math. Gen. 15,2599 (1982)

[5] C.V. Manakov, V.E. Zakharov, L.A. Bordag, et al., Phys. Lett. 63A, 205 (1977).

[6] R.P. Feynman, In: Progress in Low Temperature Physics, Vol. 1, (ed. C.J. Gorter), p. 17, North-Holland, Amsterdam, (1955).

[7] N.G. Berloff, J. Phys. A: Math. Gen. 37, 1617-1632 (2004).

[8] N.G. Berloff, private communication.

[9] S. Komineas, N. Papanicolaou, Phys. Rev. A, 68, 043617 (2003).

[10] R.G. Scott, F. Dalfovo, L.P. Pitaevskii, and S. Stringari, Phys. Rev. Lett. 106, 185301 (2011).

[11] F.D.V. Haldane, Phys. Rev. Lett. 47, 1840 (1981).

[12] L.P. Pitaevskii, arXiv:1311.4693,

[13] J. Brand, W.P. Reinhardt, Phys. Rev. A 65, 043612 (2002).

[14] M.J.H. Ku, Wenjie Ji, B. Mukherjee, et al., arXiv:1402.7052.

[15] S. Donadello, S. Serafini, M. Tylutki, et al., arXiv:1404.4237. 